\title{
The Role of Bone Scintigraphy in Determining Spinal Fusion after Spinal Stabilisation Surgery
}

\author{
Ozgur DEMIR ${ }^{1}$, Fatih Ersay DENIZ1, Erol OKSUZ¹, Serdar Savas GUL², Osman DEMIR ${ }^{3}$
}

${ }^{1}$ Gaziosmanpaşa University, Department of Neurosurgery, Tokat, Turkey

${ }^{2}$ Gaziosmanpaşa University, Department of Nuclear Medicine, Tokat, Turkey

${ }^{3}$ Gaziosmanpaşa University, Department of Biostatistics, Tokat, Turkey

This study has been presented as an oral presentation at the $32^{\text {nd }}$ Scientific Congress of Turkish Neurosurgical Society, between 20 and 24 April 2018 , at Antalya, Turkey.

Corresponding author: Ozgur DEMIR cerendemir40@gmail.com

\section{ABSTRACT}

AIM: To evaluate the usefulness of bone scintigraphy in spinal fusion surgery.

MATERIAL and METHODS: This retrospective study included 21 patients who had undergone previous anterior or posterior spinal fusion procedures, or both. Implant failure, fusion failure and adjacent segment disease were the evaluated pathological parameters. Scintigraphic data from all patients were evaluated with intraoperative observational data, radiological data and clinical data.

RESULTS: Radiological evaluation revealed adjacent segment disease in 5 patients (23.8\%), implant failure in 2 (9.5\%), and fusion failure in 1 (4.8\%). Scintigraphic evaluation of operating segments revealed pseudo-fusion in 3 patients (14.3\%) and fusions in 18 (85.7\%). Reoperations were performed in 9 patients (42.9\%): in 5 (23.8\%) because of adjacent segment disease, and in 4 (19.0\%) because they requested removal of the implants. Two patients $(9.5 \%)$ with implant failure did not undergo reoperation because their scintigraphic data were consistent with fusion and they were almost symptom free, with lower Visual Analogue Scale (VAS) scores. The VAS scores of the rest of the patients were significantly reduced after the reoperations $(p<0.001)$.

CONCLUSION: Bone scintigraphy may be helpful for surgeons in planning appropriate surgical revision strategy by giving proper data about spinal fusion at least one year after the initial surgery.

KEYWORDS: Scintigraphy, Spinal fusion, Back pain

\section{INTRODUCTION}

$\mathrm{R}$ ecurrence of pain is still challenging problem after spinal stabilisation operations. The surgeons want ensure spinal fusion in operations. Determining the occurrence of spinal fusion is important for defining surgical or nonsurgical treatment strategies $(5,24)$. In general, spinal fusion takes up to one year after the spine surgery to be complete. Therefore, surgeons generally do not plan further surgery during this period. In cases of obvious failure of spinal construct and instrumentation, reoperation can be planned sooner (10).
It is still hard to detect bone fusion one year after the fusion surgery. Diagnosis of fusion is very difficult, and depends on clinical and radiological findings (21). Clinical symptoms for pseudounion are often nonspecific, pain is the most common symptom (13). There are several studies in the literature about the imaging sensitivity and specificity of some radiological studies for diagnosis of spinal fusion. Plain radiographs, computed tomography (CT), and magnetic resonance imaging (MRI) are the studies used most commonly to confirm spinal fusion. However, studies have failed to provide enough data for determining spinal fusion (9). Recently, single photon emission
Ozgur DEMIR (10: 0000-0002-9450-3906

Fatih Ersay DENIZ : (1) : 0000-0001-6519-9741
Erol OKSUZ (1) : 0000-0002-3831-0309

Serdar Savas GUL (1) : 0000-0003-4822-2588
Osman DEMIR (D) : 0000-0002-1322-2716 
computed tomography (SPECT) combined with CT (SPECT/ CT) has become one of the most popular studies used to show fusion $(16,22)$. Bone scintigraphy has rarely been used to show pseudarthrosis $(12,16)$. In our retrospective study, we tried to show the efficacy of bone scintigraphy for determining bone fusion after spinal fusion surgery.

\section{MATERIAL and METHODS}

This retrospective study included data from 21 patients who had undergone different spinal instrumentation and fusion surgery. All patients were admitted to our clinic because of recurrence of pain. Their medical records-including plain radiographs, CT/MRI results, bone scintigraphic findings, operation reports, and Visual Analogue Scale (VAS) scores were analysed retrospectively.

Mean age at the time of the first surgery was 54.76 years (range, $14-80$ years). Nine patients (42.9\%) were male and 12 $(57.1 \%)$ were female. The first operation consisted of spinal fusion and instrumentation in all patients. The indications for primary surgery were spinal stenosis (66.7\%) and spinal trauma (33.3\%). All patients had pain, which developed after a long symptom-free period. The mean length of the symptomfree period was 2.76 years (range, 1-7 years).

Besides conventional radiological studies, bone scintigraphy was performed for all the patients to evaluate spinal fusion after the patients complained of pain. Bone scans were performed with a dual-head gamma camera (Symbia E; Siemens, Erlangen, Germany) in this study. Anterior and posterior wholebody scans were performed simultaneously with low-energy, high-resolution parallel hole collimation with a speed of $15 \mathrm{~cm} /$ min. A $256 \times 1024$-pixel matrix was used. Whole-body bone scans up to $800 \mathrm{MBq}$ (range, $650-800 \mathrm{MBq}$ ) of technetium99m-labelledmethylene diphosphonate (Tc-99m MDP) were acquired during the early part of the injection and 3 hours after injection. Tc-99m MDP radiopharmaceutics incorporation into the bone depends on perfusion and rate of new bone formation. Tc-99m MDP and its analogs localize to bone by chemisorption to the surface of hydroxyapatite crystals. Imaging usually is performed two to fours after injection. The patient's images were examined for the presence of fusion activity in the early and late periods. Results were read by an experienced nuclear medicine physician. All cases were visually assessed for any asymmetry of tracer uptake in the suspected location. Diffused or locally increased uptake in the affected sites shown in any of the three phases as compared with the same of the contralateral side was considered as a positive sign, while symmetrical uptake as a negative sign. A lack of fusion activity in the early and late periods indicated that the fusion process was completed. With pseudoarthrosis, there was continued motion with impact loading on the adjacent bone surfaces. Impact loading on the bone activates the osteoblast, which results in increased uptake of Tc-99m MDP.

Surgical records of the patients were analysed retrospectively. Surgical data were obtained from observation of movement in the surgical area. Segments in which fusion was expected but that had been shaken from pedicular screws or spinous processes were examined, and presence or absence of movement was recorded.

We collected data from conventional radiological, nuclear medicine and observational intraoperative findings and compared them with each other. One-year follow-up of clinical and radiological findings was also included in in this comparison. Pathological findings were classified as adjacent segment disease, fusion failure and implant failure.

We also evaluated the VAS scores of all the patients from the initial assessment and follow-up. This enabled us to evaluate pain intensity of all the patients by marking a $10-\mathrm{cm}$ line anchored with terms. Results are examined by statistical analysis. Values are expressed as mean \pm SD. For time and group factors, Repeated Measures ANOVA was used. A $\mathrm{p}$-value $<0.05$ was considered significant. Analyses were performed using SPSS 19 (IBM SPSS Statistics 19, SPSS inc., an IBM Co., Somers, NY).

\section{RESULTS}

We detected some pathological findings on conventional radiological studies. We found implant failure in 2 patients $(9.5 \%)$, adjacent segment disease in 5 (23.8\%), and fusion failure in 1 (4.8\%). In both patients with implant failure, broken screws were the only cause. Causes of the adjacent segment disease were spondylolisthesis on the upper level in two patients $(9.5 \%)$, compression fracture on the upper level in one patient (4.8\%), and spinal stenosis on the upper level in two patients $(9.5 \%)$. The cause of the one case of fusion failure was increased compression fracture and oedema in the vertebral corpus, according to radiological studies.

We compared radiological findings of all the patients with their bone scintigraphy findings. Bone scintigraphy showed completed fusion processes at the operational sites in both patients with implant failure and continuing fusion processes at the operational area in the patient with increased compression fracture. In four of the five patients with adjacent segment disease, bone scintigraphy findings were compatible with completed fusion processes at the operational sites; in the fifth patient, bone scintigraphy showed a continuing fusion process at the operational site. In the patient with fusion failure, bone scintigraphy revealed pseudo-fusion at the operational site (Table I).

The lack of fusion activity shown by bone scintigraphy in the early and late periods at the operational sites showed that the fusion process was completed (Figure 1A, B). The presence of fusion activity in the early and late periods showed continuation of the fusion process at the operational site (Figure 2A, B). Bone scintigraphic results of all the patients revealed that three $(14.3 \%)$ had pseudo-fusions and 18 $(85.7 \%)$ had completed fusions at the operational sites. Of the patients with pseudo-fusions, one (4.8\%) had adjacent segment disease, one (4.8\%) had fusion failure, and one $(4.8 \%)$ had implant failure. The patient with adjacent segment disease underwent reoperation. Surgical findings were compatible with scintigraphic findings: fusion was incomplete 
Demir O. et al: Bone Scintigraphy for Spinal Fusion

Table I: General Characteristic of Variables

\begin{tabular}{lc}
\hline Characteristic & Value $\mathrm{n}(\%)$ \\
\hline Scintigraphy & \\
\hline Non-fusion & $3(14.3)$ \\
Fusion & $18(85.7)$ \\
\hline Radiology & $1(4.8)$ \\
\hline Fusion failure with increased & \\
Compression fracture and kyphosis & $5(23.8)$ \\
Adjecent segment disease & $2(9.5)$ \\
Implant failure with broken screw & $13(61.9)$ \\
No pathology & \\
\hline Reoperation & $2(9.5)$ \\
\hline Rejected & $5(23.8)$ \\
Yes due to pathology & $4(19.0)$ \\
Yes due to patient request & $10(47.6)$ \\
No & Mean \pm SD \\
\hline & $5.19 \pm 1.86$ \\
\hline Initial VAS ( $n=21)$ & $2.16 \pm 0.90$ \\
\hline Follow-up VAS $(n=19)$
\end{tabular}

at the operational site. Patients with fusion failure and implant failure did not accept the offers of reoperation.

Of the patients with completed fusions in the operational sites, two $(9.5 \%)$ exhibited implant failure and 16 (76.2\%) exhibited no radiological abnormalities. The patients with implant failure did not undergo revision surgery. Four (19.0\%) patients with no radiological abnormalities underwent reoperation because they requested removal of the implants. These implants were simply taken out in the reoperation. The scintigraphic data of these patients were also compatible with surgical findings: data: fusions at the operational sites were complete in all these patients.

As mentioned previously, five patients (23.8\%) underwent reoperation because of adjacent segment disease, and four $(19.0 \%)$ because they requested removal of implants. We checked and documented the expected fusions at the operational sites during these operations. We compared the patients' surgical data with their scintigraphy data; all the surgical findings were compatible with scintigraphy findings. At surgery, we detected completed fusion at the surgical areas in the four patients who requested removal of implants; this

SD: Standart deviation, VAS: Visual analogue scale.
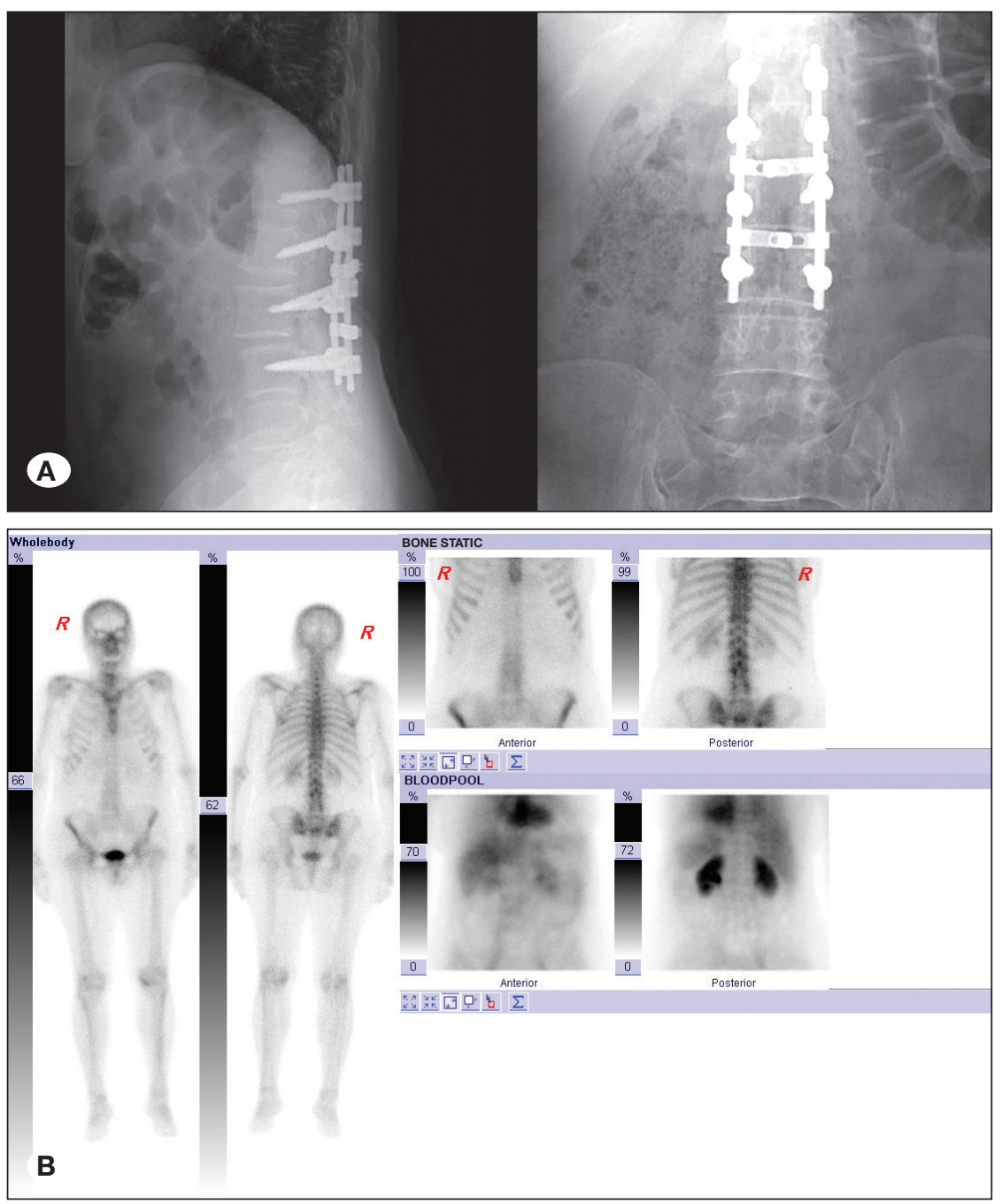

Figure 1: Images that showed no fusion activity. A) Plain radiography showed posterior lumbar instrumentations associated with stabilisation and fusion surgery. B) Bone scintigraphy showed no increased activity in the early period and bone phase. 
finding was verified by bone scintigraphy. Therefore, it was feasible to remove these items of these patients. According to bone scintigraphy findings, fusion was complete in four of five patients with adjacent segment disease. Surgical findings of the same patients also verified completion of fusion. For this reason, the previous implants of these patients were removed and new ones were placed in the adjacent segment area. In 1 of 5 patients with adjacent segment disease, the fusion process was not complete, according to bone scintigraphy and surgical findings. There was movement at the operational area where fusion was expected. Therefore, we did not take out the original implants, but we added new ones to the adjacent segment area.

We evaluated all patients at the one-year follow-up. Two patients-one with implant failure and a broken screw, and one with fusion failure refused the recommended treatment and did not participate in further routine follow-up. Bone scintigraphy findings in these patients were consisted with nonfusion at the operational sites. The nine patients who underwent reoperation had no radiological abnormalities or clinical complaints in the follow-up. No additional pathological process was detected in any patient in whom implants were removed or renewed.

Eleven patients who did not undergo reoperation were evaluated according to follow-up records. In nine of these patients, radiological studies revealed no pathological findings and the scintigraphic studies confirmed completion of the fusion process. In the follow-up of these patients, we found no clinical and radiological pathological processes. The other two patients demonstrated implant failure with broken screws. We did not reoperate on these patients because they were almost symptom free. In the follow-up, these patients remained almost symptom free, and we did not detect any pathological movement at the operational sites in the hyperextension and flexion radiographs.

We evaluated VAS scores of all patients in the initial assessment and follow-up. The patients with adjacent segment disease had the highest VAS scores in the initial assessment $(p<0.001)$. These scores decreased significantly after reoperations $(p<0.001)$. The patient with fusion failure had also a high VAS score initially, but we could not evaluate it statistically because of the insufficient number of patients.
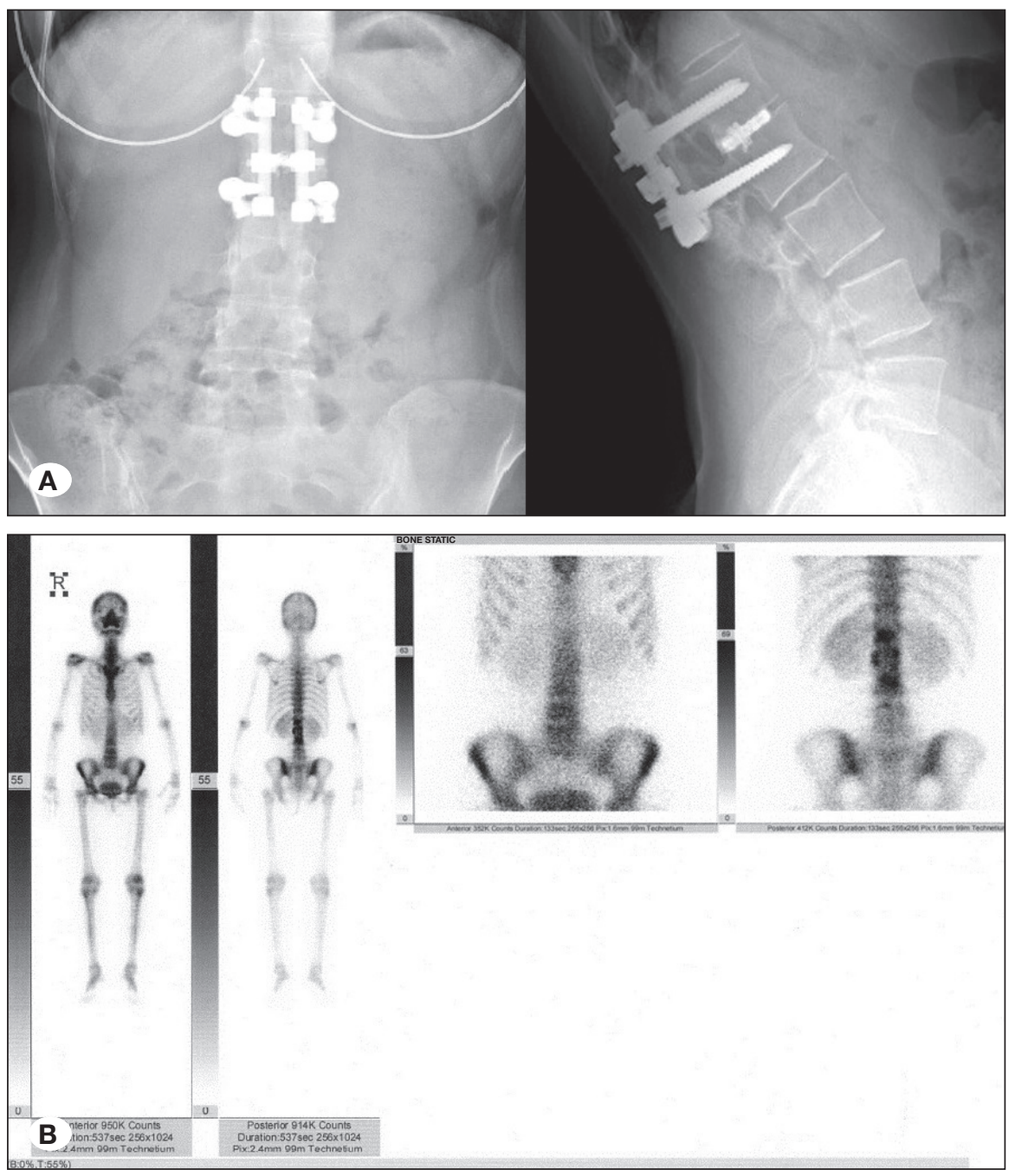

Figure 2: Images that showed fusion activity. A) Plain radiography showed posterior lumbar and inter-vertebral instrumentations associated with stabilisation and fusion surgery. B) Bone scintigraphy showed increased activity in the early period and bone phase. 
The patients with implant failure had the lowest VAS scores in the initial and follow-up assessments $(p<0.001)$, and the scores in the initial assessment did not change significantly in the follow-up assessment $(p=0.302)$. For the patients with no pathological findings, VAS scores were moderate in the initial assessment and decreased significantly in the follow-up assessment $(p<0.001)$. Patients who underwent reoperation because of pathological problems had the highest VAS scores $(p<0.001)$ in the initial assessment. Their scores decreased significantly after the reoperations $(p<0.001)$. In patients who underwent reoperation for removal of implants, VAS scores also were initially moderate and decreased significantly after the operations $(p=0.001$; Table II).

\section{DISCUSSION}

The most common complication in our study was adjacent segment disease. Clinical studies have already shown that after stabilisation and fusion surgery, load and movement on adjacent segments are increased. This situation can cause many pathological conditions, including spondylolisthesis, herniated nucleus pulposus, spinal stenosis, and compression fracture in the adjacent segment $(11,14)$. In our study, spinal stenosis and spondylolithesis were the most common abnormalities. Vertebral compression fracture was also detected in our study. The incidence of adjacent segment disease ranges from $2 \%$ to $18.5 \%$ in the literature (11); the incidence in our study seems higher. This result may have occurred because we selected patients who had pain symptom after a long symptom-free period.

The second most common complication in our study was implant failure. Causes of implant failure generally depend on surgical technique, implant quality, the patient and the diagnosis. The incidence of implant failure in the literature ranged from $9 \%$ to as high as $60 \%(3,15)$; the incidence in our study was $9.5 \%$. Fusion failure was the least common complication in our study. Fusion failure is caused by poor bone healing, which may be due to the patient's tissues, bone graft quality and excessive motion across the fusion site. According to the literature, the rates of fusion failure range from $5 \%$ to $35 \%$ after spine fusion surgeries (1), whereas the incidence in our study was $4.8 \%$. These results seem to be consistent with the literature.

With regard to removal of spinal implants in the case of completed fusion without any new complications, there are no well-defined data. Some researchers tried to find answer the question of whether spinal implants continue to produce pain even though the fusion process is complete. According to the literature, spinal implants may be a source of pain, according to some theories. Mechanical impingement, prominence and stress transfer are the major reasons in theories of how pain is generated from spinal implants. In addition, immune reaction to corrosion debris from the metal of the implant is one of the most popular theories. Metal can corrode through mechanical movement or through biological interaction. Metal ions liberated by this corrosion can initiate local inflammation $(4,24,25)$. We did not find any data about metal corrosion in the medical records of the patients who undergone reoperations.

In our study, four patients underwent reoperation to remove the implants. Bone scintigraphy demonstrated completed bone fusion at the operational sites, and radiological studies showed no additional pathological processes. According to medical data of these patients, no abnormal movement was detected in operational sites. Surgical observations of the patients were consisted with completed bone fusion. After establishing these findings, we removed the implants as requested. The VAS scores of these patients decreased significantly after their implants were removed $(p<0.001)$. Additional follow-up radiological studies showed no pathological findings. Our results may support the theory in the literature that spinal implants can be a source of pain even after the fusion is complete. Bone scintigraphy successfully demonstrated bone fusion in all four patients. For this reason, we can recommend the use of scintigraphy before surgical removal of implants.

The other important issue in this study is management of patients with implant failure. Some researchers have tried to show that implant fractures due to implant failure can occur even in the case of fusion $(6,19)$. They also presented some

Table II: Distribution of VAS Scores by Radiology

\begin{tabular}{|c|c|c|c|}
\hline \multirow[t]{2}{*}{ Radiology } & $\begin{array}{l}\text { Initial VAS score } \\
(n=21)\end{array}$ & $\begin{array}{l}\text { Follow-up VAS score } \\
\qquad(n=19)\end{array}$ & \multirow{2}{*}{$p_{1}$} \\
\hline & Mean \pm SD & Mean \pm SD & \\
\hline Fusion failure with increased compression fracture and kyphosis & $8.00 \pm$. & - & - \\
\hline Adjecent segment disease & $7.00 \pm 0.71^{a}$ & $2.60 \pm 0.89$ & $<0.001$ \\
\hline No pathology & $4.69 \pm 1.44^{b}$ & $2.08 \pm 0.90$ & $<0.001$ \\
\hline$\underline{p_{2}}$ & $<0.001$ & 0.324 & \\
\hline Total & $5.19 \pm 1.86$ & $2.16 \pm 0.9$ & $<0.001$ \\
\hline
\end{tabular}

$\boldsymbol{p}_{\mathbf{1}}$ : Within-Subjects comparison, $\boldsymbol{p}_{2}$ : Between-Subjects comparison, $\boldsymbol{a}, \boldsymbol{b}$ : In the same column, different superscripts indicate statistical significance. 
theories to support this thesis. One of these theories is that the residual implants provide sufficient stability for bone fusion. Another is that the solid fusion mass can cause metal fatigue in multiple areas by forcing the implants to bend. These studies also emphasised that symptoms of the patients are very important $(6,19)$. The patients should not have de novo symptoms. Two of our patients had implant failures detected in radiological studies. The patients had broken screws without de novo symptoms. They were almost symptom free, with lower VAS scores in the initial evaluation. Bone scintigraphy of these patients demonstrated that bone fusion at the operational sites was complete. Hyperextension and flexion plain radiographs showed no movement at the operational sites in the initial and follow-up evaluations. There were also no significant differences between follow-up and initial VAS scores $(p=0.302)$. Therefore, our results support the thesis that implant failure can occur even in the case of completed bone fusion. In case of implant failure, it is important before reoperation to confirm absence of de novo symptoms and presence of bone fusion. Bone scintigraphy can be used to confirm bone fusion in the case of implant failure.

The frequency of spinal fusion surgery has increased significantly in recent years. The aim of spinal instrumentation is to provide additional spinal stability while bone fusion is occurring. The many causes of pain identified after spinal fusion include adjacent segment disease, implant failure and fusion failure. Implants also should come to mind as a source of pain. Before surgeons decide on reoperation for ongoing pain after spinal fusion surgery, bone fusion should be assessed because the strategy of reoperation depends on whether fusion is complete (8).

Certain radiological methods are commonly used to assess spinal fusion. Imaging is generally performed in the postoperative period for several reasons: to confirm positioning and integrity of hardware, to detect expected complications, to detect subsequent new pathological problems, and to assess progression of bone fusion. Conventional radiological techniques, such as $\mathrm{CT}$, MRI, plain radiography, and dynamic radiography, are generally sufficient for all aims except assessing bone fusion $(2,7,17,18)$. Limitations of these techniques to assess bone fusion generally concern metallic artefact and radiolucency. Nuclear medicine, including bone scintigraphy, is also used to assess fusion sites. In this technique, the fused segment should appear cold after 6 to 12 months. Bone scintigraphy has been found to be helpful especially in the cases in which MRI and CT cannot be performed or are not feasible because of the presence of metallic hardware.

There is also some criticism in the literature about the use of bone scintigraphy. One problem is that radionuclide scans may continue to be positive more than one year as a result of continued normal bone fusion. Another is that very focal intense activity may reflect pseudo-fusion, whereas diffuse intense activity may reflect spine fusion $(16,20,23)$. We aimed to show the efficacy of bone scintigraphy in the diagnosis of bone fusion. In our study, the success rate of bone scintigraphy was $100 \%$ in patients who continued treatment at our clinic. The scintigraphic results were confirmed by radiological and clinical evaluation in the initial and follow-up examinations.
Surgical observations were also used for confirmation in the patients who underwent reoperation. All these data supported the accuracy of the scintigraphic results. This result may be due to patient selection: we used bone scintigraphy in the patients who had undergone initial surgery at least one year previously. Bone scintigraphy may give misleading results less than one year after surgery because of technical causes; therefore, we recommend the use of bone scintigraphy in determining spinal fusion one year after the spinal fusion surgery. In our view, bone scintigraphy appears to be an important support to clinical and radiological evaluations for determining bone fusion.

\section{Limitations}

In our retrospective study, the number of cases was small, and length of follow-up was short. We recommend long-term follow-up of a cohort of patients who have undergone spinal fusion and normal controls to provide further evidence.

\section{- REFERENCES}

1. Berjano $P$, Langella F, Damilano $M$, Pejrona M, Buric J, Is- mael M, Villafane $\mathrm{JH}$, Lamartina C: Fusion rate following extreme lateral lumbar interbody fusion. Eur Spine J 24:369-371, 2015

2. Brodsky AE, Kovalsky ES, Khalil MA: Correlation of radiologic assessment of lumbar spine fusions with surgical exploration. Spine 16:261-265, 1991

3. Christensen FB, Dalstra M, Sejling F, Overgaard S, Bunger C: Titanium-alloy enhances bone-pedicle screw fixation: Mechanical and histomorphometrical results of titanium-alloy versus stainless steel. Eur Spine J 9:97-103, 2000

4. DePalma MJ, Ketchum JM, Saullo TR: Etiology of chronic low back pain in patients having undergone lumbar fusion. Pain Med 12:732-739, 2011

5. Diwan AD, Parvartaneni $H$, Cammisa F: Failed degenerative lumbar spine surgery. Orthop Clin North Am 34:309-324,2003

6. Erwin WD, Dickson JH, Harrington PR: Clinical review of patients with broken Harrington rods. J Bone Jt Surg Am 62:1302-1307, 1980

7. Fogel GR, Toohey JS, Neidre A, Brantigan JW: Fusion assessment of posterior lumbar interbody fusion using radiolucent cages: X-ray films and helical computed tomography scans compared with surgical exploration of fusion. Spine J 8:570-577,2008

8. Goldstein C, Drew B: When is a spine fused? Injury 42:306313, 2011

9. Gruskay JA, Webb ML, Grauer JN: Methods of evaluating lumbar and cervical fusion. Spine J 14:531-539, 2014

10. Herkowitz HN, Sidhu KS: Lumbar spine fusion in the treatment of degenerative conditions: Current indications and recommendations. J Am Acad Orthop Surg 3:123-135, 1995

11. Hilibrand AS, Robbins M: Adjacent segment degeneration and adjacent segment disease: The consequences of spinal fusion? Spine J 4:190-194, 2004

12. Kim PE, Zee CS: Imaging of the postoperative spine: Cages, prostheses, and instrumentation. In: Van Goethem JWM, Van Den Hauwe L, Parizel PM (eds), Spinal Imaging: Diagnostic Imaging of the Spine and Spinal Cord. Berlin: Springer, 2007:397-413 
Demir O. et al: Bone Scintigraphy for Spinal Fusion

13. Kornblum MB, Fischgrund JS, Herkowitz HN, Abraham DA, Berkower DL, Ditkoff JS: Degenerative lumbar spondylolisthesis with spinal stenosis: A prospective long term study comparing fusion and pseudarthrosis. Spine (Phila Pa 1976) 29:726-734, 2004

14. Levin DA, Hale JJ, Bendo JA: Adjacent segment degeneration following spinal fusion for degenerative disc disease. Bull NYU Hosp Jt Dis 65:29-36,2007

15. McLain RF, Sparling E, Benson DR: Early failure of shortsegment pedicle instrumentation for thoracolumbar fractures. A preliminary report. J Bone Joint Surg Am 75:162-167, 1993

16. McMaster MJ, Merrick MV: The scintigraphic assessment of the scoliotic spine after fusion. J Bone Joint Surg Br 62:6572,1980

17. Pai VS, Hodgson B: Assessment of bony union following surgical stabili- sation for lumbar spondylolysis: A comparative study between radiography and computed tomography. J Orthop Surg (Hong Kong) 14:17-20, 2006

18. Port JD, Pomper MG: Quantification and minimization of magnetic sus-ceptibility artifacts on GRE images. J Comput Assist Tomogr 24:958-964,2000

19. Przybyla AS, Skrzypiec D, Pollintine P, Dolan P, Adams MA: Strength of the cervical spine in compression and bending. Spine (Phila Pa 1976) 32:1612-1620,2007
20. Rager O, Schaller K, Payer M, Tchemin D, Ratib O, Tessitore E: SPECT/CT in differentiation of pseudarthrosis from other causes of back pain in lumbar spinal fusion: Report on 10 consecutive cases. Clin Nucl Med 37:339-343, 2012

21. Rutherford EE, Tarplett LJ, Davies EM, Harley JM, King LJ: Lumbar spine fusion and stabilization: Hardware, techniques and imaging appearances. Radiographics 27:1737-1749,2007

22. Schiesser M, Stumpe KD, Trentz O, Kossmann T, Von Schulthess GK: Detection of metallic implant-associated infections with FDG PET in patients with trauma: Correlation with microbiologic results. Radiology 226:391-398, 2003

23. Sumer J, Schmidt D, Ritt P, Lell M, Forst R, Kuwert T, Richter R: SPECT/CT in patients with lower back pain after lumbar fusion surgery. Nucl Med Commun 34:964-970,2013

24. Waguespack A, Schofferman J, Slosar P, Reynolds J: Etiology of long-term failures of lumbar spine surgery. Pain Med 3:1822,2002

25. Zotti MG, Brumby-Rendell OP, McDonald B, Fisher T, Tsimiklis C, Yoom WW, Osti OL: The outcome of pedicle screw instrumentation removal for ongoing low back pain following posterolateral lumbar fusion. J Spine Surg 1:50-56,2015 